A. Bremner

Nagoya Math. J.

Vol. 102 (1986), 101-115

\title{
ON ELLIPTIC SURFACES OF MORDELL-WEIL RANK 4
}

\author{
ANDREW BREMNER
}

\section{$\S 1$.}

Let $f: E \rightarrow \boldsymbol{P}^{\prime}(C)$ be an elliptic fibration with non-constant $j$-invariant, and possessing a section $\sigma_{0}$. The group (s) of sections of $f$ is then naturally identified with the group of points defined over a function field $C(u)$ of the generic fibre of $f$.

Take as generic fibre the Weierstrass equation

$$
y^{2}=4 x^{3}-g_{2} x-g_{3}, \quad g_{2}, g_{3} \in C(u)
$$

so that $(S)$ is isomorphic to the group of $C(u)$-rational solutions of (1), with the zero section $\sigma_{0}$ corresponding to the point at infinity. By abuse of notation we shall denote this latter group also by $\$ \$$.

The geometric genus $p_{g}$ of the elliptic surface $E$ is determined by a knowledge of the singular fibres of $f$ (see Kodaira [4] or 2.5 of Cox and Zucker [3]); and when $p_{g}=0$ then the rank $r$ of $\$ s$ is easily determined by a formula of Shioda [7]. It turns out that if $p_{g}=0$ then the model (1) may be chosen so that $g_{2}$ and $g_{3}$ are respectively quartic and sextic polynomials in $C[u]$; and for generic $g_{2}, g_{3}$ the rank of (S) equals 8 .

Let $P=\left(x_{0}, y_{0}\right) \in \mathbb{B}$; define $P$ to be at most quadratic if

(i) $x_{0}, y_{0} \in C[u]$

(ii) the degree of $x_{0}$ in $u$ is at most 2 .

Then Schwartz [5] proves that for almost all choices of $g_{2}, g_{3}$, it is the case that $(5)$ is generated by the points of (1) which are at most quadratic; and conjectures that this is still true for all choices of $g_{2}, g_{3}$.

We consider in this paper the subset of such surfaces where there is a rational section of order 2 . In particular, we take as model for the generic fibre the surface

$$
y^{2}=x\left(x^{2}+m x+n\right)
$$

with $m, n \in C[u]$ of degrees at most 2,4 , respectively. The rank of \& for

Received November 26, 1984. 
generic $m, n$, respectively quadratic, quartic, has dropped to 4 . We show that under some weak restrictions on $m, n$ then the rank is precisely 4 and $B S$ is indeed generated by the at most quadratic points. Further, the restrictions on $m, n$ can be removed to show that if the rank of \&s does equal 4 , then 85 is generated by the at most quadratic points.

Whereas Schwartz uses a specialization technique, resulting in 'almost all', the method here is an application of some careful estimates on heights. It is not without possibility that similar methods will resolve the original conjecture of Schwartz, but the intricate computations seem a little daunting.

In the final section, we make some remarks about the field of definition of (\$).

$\S 2$.

We are considering the elliptic surface over $C(u)$

$$
E: y^{2}=x\left(x^{2}+m x+n\right)
$$

with

$$
m, n \in C[u], \quad \operatorname{deg}(m) \leq 2, \quad \operatorname{deg}(n) \leq 4 .
$$

The group of $C(u)$ points on (2) will be denoted by (5).

Lemma 1. The surface (2) has Mordell-Weil rank 4 over $C(u)$ when the following hold:

(i) $n$ and $m^{2}-4 n$ are of degree 4

(ii) $n$ and $m^{2}-4 n$ are squarefree elements of $C[u]$.

Proof. This will be shown in the course of the derivation below of a system of generators for (\$)/2\&s. Henceforth, we shall hypothesize that $m, n$ satisfy conditions (4), (5).

The bulk of this section is devoted to showing that it is possible to find a set of points on $E$, nearly all of which (29 out of 32 , to be precise!) are at most quadratic points whose cosets form a full set of representatives for $\& / 2 \&$. The verification is by descent, following Cassels, Ellison, Pfister [2].

Consider $E$ in conjunction with the curve

$$
E^{\prime}: Y^{2}=X\left(X^{2}-\frac{m}{2} X+\frac{1}{16}\left(m^{2}-4 n\right)\right) \text {. }
$$


There are 2-isogenies $\nu, \nu^{\prime}$ whose composition is multiplication by 2 , as follows:

where

$$
\Gamma \stackrel{\nu^{\prime}}{\longrightarrow} \Gamma^{\prime} \stackrel{\nu}{\longrightarrow} \Gamma
$$

$$
\begin{aligned}
& \nu(X, Y)=\left(\frac{Y^{2}}{X^{2}}, \frac{Y}{X^{2}}\left(X^{2}-\frac{1}{16}\left(m^{2}-4 n\right)\right)\right) \\
& \nu^{\prime}(x, y)=\left(\frac{y^{2}}{4 x^{2}}, \frac{y}{8 x^{2}}\left(x^{2}-n\right)\right) .
\end{aligned}
$$

Suppose first $P$ is a point of $E$, defined over $C(u)$. Then

$$
P=\left(\frac{\Delta R^{2}}{S^{2}}, \frac{\Delta R T}{S^{3}}\right)
$$

where, $\Delta, R, S \in C[u], \Delta$ squarefree, $(R, S)=1$, and

$$
T^{2}=\Delta R^{4}+m R^{2} S^{2}+\frac{n}{\Delta} S^{4} .
$$

When (8) holds, say that $P$ belongs to $\Delta$. By appropriate multiplication of $R$ by an element of $C$, the leading coefficient of $\Delta$ may be assumed if necessary to equal 1 .

For local solvability of (2), it is necessary that $n$ be divisible by $\Delta$. By hypothesis (5), $n$ splits into four distinct linear factors over $C$, and there are accordingly $2^{4}$ possibilities for $\Delta$. However, if has $\Delta$ odd degree in $u$, then since the right hand side of (9) must have even degree, there is cancellation between leading coefficients of $\Delta R^{4}$ and $(n / \Delta) S^{4}$. In particular, they have the same degree. But this is contradictory to $\Delta$ having odd degree.

Thus, there are at most $2^{3}$ possibilities for $\Delta$. If $\Delta \in C$ or $(n / \Delta) \in C$ then there certainly do exist solutions of (9) with $S=0, R=0$ respectively. Suppose now $\Delta$ is quadratic, $\Delta=a_{1} u^{2}+a_{2} u+a_{3}, a_{i} \in C$. Further, put $m=b_{1} u^{2}+b_{2} u+b_{3}, n / \Delta=c_{1} u^{2}+c_{2} u+c_{3}, b_{i}, c_{i} \in C$. Then from (9).

$$
\begin{gathered}
T^{2}=u^{2}\left(a_{1} R^{4}+b_{1} R^{2} S^{2}+c_{1} S^{4}\right)+u\left(a_{2} R^{4}+b_{2} R^{2} S^{2}+c_{2} S^{4}\right) \\
+\left(a_{3} R^{4}+b_{3} R^{2} S^{2}+c_{3} S^{4}\right) .
\end{gathered}
$$

If $R / S \in C$, then the right hand side of (10) is a perfect square in $C[u]$ if and only if

$$
\begin{aligned}
& \left(a_{2} R^{4}+b_{2} R^{2} S^{2}+c_{2} S^{4}\right)^{2} \\
& \quad=4\left(a_{1} R^{4}+b_{1} R^{2} S^{2}+c_{1} S^{4}\right)\left(a_{3} R^{4}+b_{3} R^{2} S^{2}+c_{3} S^{4}\right)
\end{aligned}
$$


Now (11) certainly does have solutions for $R / S$, and so we can construct $2^{3}$ points of $E$ corresponding to the $2^{3}$ possibilities for $\Delta$, whose cosets are representatives for the group $\mathbb{S} / \nu\left(\mathbb{S}^{\prime}\right)$. This latter remark is by standard arguments; see for example Birch and Swinnerton-Dyer [1].

However, consider more closely solutions of (11). There certainly are four such solutions in $C$ for the ratio $R^{2} / S^{2}$, and these solutions are non-zero and finite since the coefficients of $R^{8}, S^{8}$ are $a_{2}^{2}-4 a_{1} a_{3}, c_{2}^{2}-4 c_{1} c_{3}$ respectively, non-zero by hypothesis (5). Moreover, the four solutions are distinct, as may be shown as follows.

The condition that (11) have a repeated root for $R^{2} / S^{2}$ is just that the discriminant $I^{3}-27 J^{2}$ of the associated quartic equation should be zero (here $I, J$ are the classical quartic invariants). But, as is straightforward to check, the invariants $I, J$ of (11) are precisely equal to the invariants of the quartic

$$
\left(b_{1} u^{2}+b_{2} u+b_{3}\right)^{2}=4\left(a_{1} u^{2}+a_{2} u+a_{3}\right)\left(c_{1} u^{2}+c_{2} u+c_{3}\right) .
$$

Hence, a repeated root at (11) implies a repeated root at (12), which is just the statement that $m^{2}-4 n$ has a square factor in $C[u]$, contradicting (5). In sum, corresponding to each quadratic $\Delta$, there exist precisely four distinct quadratic points on $\Gamma$ with first coordinate of type $c \Delta, c \in C$.

Additionally, each such set of four points of \&s corresponds to distinct cosets in $\mathbb{S} / 2 \circlearrowleft 5$. For arguing directly, consider the points $\left(\Delta R^{2}, \Delta R T\right)$, $\left(\Delta R^{\prime 2}, \Delta R^{\prime} T^{\prime}\right), R, R^{\prime} \in C$. Their sum on $E$ is the point $Q$ with first coordinate $\left(\left(R T^{\prime}-R^{\prime} T\right) /\left(R^{2}-R^{\prime 2}\right)\right)^{2}$. Now in order that $\left(\alpha^{2}, \alpha \beta\right)$ in $\mathbb{S}$ actually lie in $2 \mathscr{S}$, it is necessary and sufficient that one of the terms $\alpha^{2} \pm \beta+$ $m / 2$ lie in $C[u]^{2}$. So $Q \in 2 \Theta s$ if and only if

$$
\Delta R^{2} R^{\prime 2}+\frac{m}{2}\left(R^{2}+R^{\prime 2}\right)+\frac{n}{\Delta} \pm T T^{\prime} \in C[u]^{2} .
$$

However,

$$
\begin{aligned}
& \left(\Delta R^{2} R^{\prime 2}+\frac{m}{2}\left(R^{2}+R^{\prime 2}\right)+\frac{n}{\Delta}+T T^{\prime}\right)\left(\Delta R^{2} R^{\prime 2}+\frac{m}{2}\left(R^{2}+R^{\prime 2}\right)+\frac{n}{\Delta}-T T^{\prime}\right) \\
& =\frac{1}{4}\left(m^{2}-4 n\right)\left(R^{2}-R^{\prime 2}\right)^{2}
\end{aligned}
$$

and since $R, R^{\prime} \in C$ and $m^{2}-4 n$ is squarefree, then (13), (14) force

$$
\Delta R^{2} R^{\prime 2}+\frac{m}{2}\left(R^{2}+R^{\prime 2}\right)+\frac{n}{\Delta} \pm T T^{\prime} \in C
$$




$$
\Delta R^{2} R^{\prime 2}+\frac{m}{2}\left(R^{2}+R^{\prime 2}\right)+\frac{n}{\Delta} \mp T T^{\prime} \in\left(m^{2}-4 n\right) C .
$$

But $\left(15^{\prime}\right)$ is impossible, because the degree of the left hand side (which is non-zero) is at most 2.

So at this stage we have 24 quadratic points of $E$ with distinct cosets

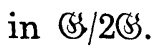

Turn now to the curve $E^{\prime}$ and in exactly the same manner select a set of $2^{3}$ points $\left(\delta r^{2} / s^{2}, \delta r t / s^{3}\right)$, each of which is at most quadratic, whose cosets are representatives for the group $\mathbb{S}^{\prime} / \nu^{\prime}(\mathbb{S})$. It follows now, from the formulae of Birch and Swinnerton-Dyer [1], for instance, that the Mordell-Weil rank of $\mathbb{S}$ and $\mathbb{S}^{\prime}$ is equal to 4 . Now $\mathfrak{D}^{\prime},(0,0) \in \mathbb{S}^{\prime}$ corresponding to $\delta=1, m^{2}-4 n$ respectively, are in the kernel of $\nu$, and the image under $\nu$ of the points representing $\mathscr{S}^{\prime} / \nu^{\prime}(\mathbb{S})$ is a group of order $2^{2}$. From (7), these points of $E$ have first coordinate $t^{2} / r^{2} s^{2}$, so are at most quadratic, and belong to $\Delta=1$. By construction, they are incongruent modulo 265. (Remark: The set of four points found previously belonging to a particular quadratic value of $\Delta$ is, modulo $2 \varangle$, just any one of these points together with its translates by means of the above set belonging to $\Delta=1$. We can obtain four representatives in $\mathbb{S} / 2 \mathbb{S}$ belonging to $\Delta=n$ by translating $(0,0)$ by the set belonging to $\Delta=1$; but now the points obtained are not quadratic.)

Theorem 2. If $P \in \mathbb{S}$, then provided $P$ does not belong to $\Delta=n$, there exists $P_{0} \in \mathbb{S}, P_{0}$ at most quadratic, with $P-P_{0} \in 2 \mathbb{S}$.

\section{$\S 3$.}

Take a general point $P$ in $(3)$. By adding to $P$ the point $(0,0)$ if necessary, we suppose that $P$ belongs to $\Delta$ where $\Delta$ is at most quadratic.

Let $P_{0} \in \mathbb{S}$ with $P \equiv P_{0} \bmod 2 \mathscr{S}$, and $P_{0}$ at most quadratic, by Theorem 2. It is the intention of this section to show that at least one of the points $P \pm P_{0}$ cannot be too 'large'. The size of a point is measured by a height function defined as follows.

Let $P=(x, y)$ with $x=p(u) / q(u), p(u), q(u) \in C[u]$; then $h(P)=$ $\max (\operatorname{deg} p(u), \operatorname{deg} q(u))$. In general, we shall use the notation $|p|$ for the degree in $u$ of the polynomial $p$.

So, suppose

$$
P=\left(\frac{\Delta R^{2}}{S^{2}}, \frac{\Delta R T}{S^{3}}\right) ; \quad P_{0}=\left(\Delta r^{2}, \Delta r t\right)
$$


with

$$
\Delta R^{4}+m R^{2} S^{2}+\frac{n}{\Delta} S^{4}=T^{2} ; \quad \Delta r^{4}+m r^{2}+\frac{n}{\Delta}=t^{2}
$$

and

$$
|\Delta|+2|r| \leq 2 \text {. }
$$

The first coordinate of $P+P_{0}$ is

$$
\left(\frac{R S t-r T}{R^{2}-r^{2} S^{2}}\right)^{2}
$$

There is from (17) the basic identity

$$
(R S t-r T)(R S t+r T)=\left(R^{2}-r^{2} S^{2}\right)\left(\frac{n}{\Delta} S^{2}-\Delta r^{2} R^{2}\right) .
$$

This can be used to choose the sign of $t$ in such a way that the highest common factor of $R S t-r T$ and $R^{2}-r^{2} S^{2}$ has degree at least half that of $R^{2}-r^{2} S^{2}$.

The proof of Theorem 7 is effected by some case-by-case lemmas.

Lemma 3. Suppose $|\Delta|=0,|r|=0$. Then, for a choice of sign, $h\left(P \pm P_{0}\right) \leq(4 / 3) h(P)+4$.

Proof. The assumptions imply $|t|=2$ from (17).

(a) $|R| \neq|S|$.

Choose the sign of $t$ according as to the remark following (20).

If $|R|>|S|$, then

$\left|R^{2}-r^{2} S^{2}\right|=2|R|,|R S t-r T| \leq 2|R|+1$ so

$$
h\left(P \pm P_{0}\right) \leq 2 \max (|R|+1,|R|)=h(P)+2 .
$$

If $|R|<|S|$, then

$\left|R^{2}-r^{2} S^{2}\right|=2|S|,|R S t-r T|=2|S|+2$ so

$$
h\left(P \pm P_{0}\right) \leq 2 \max (|S|+2,|S|)=h(P)+4 .
$$

(b) $|R|=|S|$, whence $|T|=2|R|+2$.

Now $|R S t|=2|R|+2=|r T|$, so at least one of the factors $R S t \pm r T$ has degree $2|R|+2$.

Put $\left|R^{2}-r^{2} S^{2}\right|=2 x, 0 \leq x \leq|R|$. Since $\left|(n / \Delta) S^{2}-\Delta r^{2} R^{2}\right|=2|R|+4$, it follows from (20) that $|R S t \pm r T|=2|R|+2$; $|R S t \mp r T|=2 x+2$. 
If $0 \leq x \leq \lambda|R|$ say, take the sign of $t$ such that $|R S t-r T|=2 x+2$; and then

$$
h\left(P \pm P_{0}\right) \leq 2 \max (2 x+2,2 x) \leq 4 \lambda|R|+4 .
$$

If $\lambda|R|<x \leq|R|$, take the sign of $t$ according as to the remark following (20). Then for $|R S t-r T|=2|R|+2$,

$$
h\left(P \pm P_{0}\right) \leq 2 \max (2|R|+2-x, x)
$$

and for $|R S t-r T|=2 x+2$,

$$
h\left(P \pm P_{0}\right) \leq 2 \max (x+2, x) .
$$

So in either case, (24), (25) imply

$$
h\left(P \pm P_{0}\right) \leq 2 \max ((2-\lambda)|R|+2,|R|+2) .
$$

From (23), (26) on taking $\lambda=2 / 3$, then

$$
h\left(P \pm P_{0}\right) \leq \frac{8}{3}|R|+4=\frac{4}{3} h(P)+4 .
$$

The result follows from (21), (22), (27).

Lemma 4. Suppose $|\Delta|=0,|r|=1,|R| \neq|S|+1$.

Then for a choice of sign, $h\left(P \pm P_{0}\right) \leq h(P)+4$.

Proof. The hypotheses imply $|t| \leq 2$. Choose the sign of $t$ according to remark.

(a) $|R| \geq|S|+2$ implies $\left|R^{2}-r^{2} S^{2}\right|=2|R|$,

$$
|T|=2|R|,|R S t-r T| \leq 2|R|+1 \text {. So }
$$

$$
h\left(P \pm P_{0}\right) \leq 2 \max (|R|+1,|R|)=h(P)+2 .
$$

(b) $|R|=|S|$ implies $\left|R^{2}-r^{2} S^{2}\right|=2|R|+2$.

$$
|T|=2|R|+2,|R S t-r T|=2|R|+3 \text {. So }
$$

$$
h\left(P \pm P_{0}\right) \leq 2 \max (|R|+2,|R|+1)=h(P)+4 .
$$

(c) $|R| \leq|S|-1$ implies $\left|R^{2}-r^{2} S^{2}\right|=2|S|+2$,

$$
|T|=2|S|+2,|R S t-r T|=2|S|+3 \text {. So }
$$

$$
h\left(P \pm P_{0}\right) \leq 2 \max (|S|+2,|S|+1)=h(P)+4 .
$$

The result follows from (28), (29), (30).

Lemma 5. Suppose $|\Delta|=0,|r|=1,|R|=|S|+1$. 
Then, for a choice of sign,

$$
h\left(P \pm P_{0}\right) \leq \max \left(\frac{4}{3} h(P)+4, \min \left(\frac{4}{3} h(P)+6,2 h(P)\right)\right) .
$$

Proof. The hypotheses imply $|t| \leq 2,|T| \leq 2|R|$.

Suppose $|T| \neq 2|R|-2+|t|$, so that $|R S t| \neq|r T|$. Then $|R S t \pm r T|$ $=\max \{2|R|-1+|t|, 1+|T|\}$ and so

$$
2|R|-1 \leq|R S t \pm r T| \leq 2|R|+1 .
$$

Since $\left|(n / \Delta) S^{2}-\Delta r^{2} R^{2}\right| \leq 2|R|+2$, then from (20), (31)

$$
2|R|-4 \leq\left|R^{2}-r^{2} S^{2}\right| \leq 2|R| \text {. }
$$

Choosing the sign of $t$ according to remark, then it follows that

$$
h\left(P \pm P_{0}\right) \leq 2|R|+2=h(P)+2 .
$$

The remaining cases are those for which $|T|=2|R|-2+|t|$. In this instance, then at least one of $R S t \pm r T$ has degree $|T|+1$; proceed as in Lemma 3(b). Put

$$
\begin{aligned}
& \left|R^{2}-r^{2} S^{2}\right|=2 x, \quad 0 \leq x \leq|R| ; \\
& \left|\frac{n}{\Delta} S^{2}-\Delta r^{2} R^{2}\right|=k, \quad 0 \leq k \leq 2|R|+2 .
\end{aligned}
$$

Then $|R S t \pm r T|=|T|+1,|R S t \mp r T|=2 x+k-|T|-1$. If $0 \leq x \leq$ $\lambda|R|$, take the sign of $t$ so that $|R S t-r T|=2 x+k-|T|-1$. Then

$$
\begin{aligned}
h\left(P \pm P_{0}\right) & \leq 2 \max (2 x+k-|T|-1,2 x) \\
& \leq 4 \lambda|R|+6-2|t| .
\end{aligned}
$$

If $\lambda|R|<x \leq|R|$, take the sign of $t$ according to remark.

If $|R S t-r T|=|T|+1$, then

$$
\begin{aligned}
h\left(P \pm P_{0}\right) & \leq 2 \max (|T|+1-x, x) \\
& \leq \max ((4-2 \lambda)|R|-2+2|t|, 2|R|) .
\end{aligned}
$$

If $|R s t-r T|=2 x+k-|T|-1$, then

$$
\begin{aligned}
h\left(P \pm P_{0}\right) & \leq 2 \max (x+k-|T|-1, x) \\
& \leq 2|R|+6-2|t| .
\end{aligned}
$$

With $\lambda=2 / 3$, then (34), (35), (36) imply 


$$
h\left(P \pm P_{0}\right) \leq \max \left(\frac{4}{3} h(P)+6-2|t|, \frac{4}{3} h(P)-2+2|t|\right) .
$$

In particular, for $|t|=1,2$ :

$$
h\left(P \pm P_{0}\right) \leq \frac{4}{3} h(P)+4
$$

The estimate for $|t|=0$, namely

$$
h\left(P \pm P_{0}\right) \leq \frac{4}{3} h(P)+6
$$

is not sufficient for our purposes when $h(P)$ is small. But in this instance, where $|t|=0,|r|=1,|R|=|S|+1,|T|=2|R|-2$, then $|R S t-r T|$ $\leq 2|R|-1,\left|R^{2}-r^{2} S^{2}\right| \leq 2|R|$ and it follows directly from (19) that

$$
h\left(P \pm P_{0}\right) \leq 2 h(P) .
$$

So when $|t|=0$, there is the estimate

$$
h\left(P \pm P_{0}\right) \leq \min \left(\frac{4}{3} h(P)+6,2 h(P)\right) .
$$

The result follows from (33), (38), (39).

Lemma 6. Suppose $|\Delta|=2$. Then for a choice of sign

$$
h\left(P \pm P_{0}\right) \leq \max \left(\frac{4}{3} h(P)-\frac{2}{3}, h(P)+2\right) .
$$

Proof. The hypothesis implies $|r|=0,|t| \leq 1$. If $|R| \neq|S|$, then it is straightforward as before to obtain the estimate

$$
h\left(P \pm P_{0}\right) \leq h(P)+2 .
$$

Suppose now $|R|=|S|$; then $|T| \leq 2|R|+1$. If $|T| \neq 2|R|+|t|$, then $|R S t| \neq|r T|$ and we apply the argument as at the start of Lemma 5 to show

$$
2|R| \leq|R S t \pm r T| \leq 2|R|+1 ; \quad 2|R|-2 \leq\left|R^{2}-r^{2} S^{2}\right| \leq 2|R| .
$$

Choosing the sign of $t$ according to remark, then it follows

$$
h\left(P \pm P_{0}\right) \leq h(P)+2 .
$$

The remaining cases are $|t|=1,|T|=2|R|+1$; and $|t|=0,|T|=2|R|$. In these cases at least one of $R S t \pm r T$ has degree $2|R|+|t|$. Put 


$$
\left|R^{2}-r^{2} S^{2}\right|=2 x, \quad 0 \leq x \leq|R| ; \quad\left|\frac{n}{\Delta} S^{2}-\Delta r^{2} R^{2}\right|=k, \quad 0 \leq k \leq 2|R|+2 .
$$

Then

$$
|R S t \pm r T|=2|R|+|t|, \quad|R S t \mp r T|=2 x+k-2|R|-|t| .
$$

If $0 \leq x \leq \lambda|R|$, take the sign of $t$ so that $|R S t-r T|=2 x+k-2|R|$ $-|t|$. Then

$$
\begin{aligned}
h\left(P \pm P_{0}\right) & \leq 2 \max (2 x+k-2|R|-|t|, 2 x) \\
& \leq 4 \lambda|R|+4-2|t| .
\end{aligned}
$$

If $\lambda|R|<x \leq|R|$, take the sign of $t$ according to remark.

If $|R S t-r T|=2|R|+|t|$, then

$$
\begin{aligned}
h\left(P \pm P_{0}\right) & \leq 2 \max (2|R|+|t|-x, x) \\
& \leq \max ((4-2 \lambda)|R|+2|t|, 2|R|) .
\end{aligned}
$$

If $|R S t-r T|=2 x+k-2|R|-|t|$, then

$$
\begin{aligned}
h\left(P \pm P_{0}\right) & \leq 2 \max (x+k-2|R|-|t|, x) \\
& \leq 2|R|+4-2|t| .
\end{aligned}
$$

Taking $\lambda=2 / 3$ then (42), (43), (44) imply (using $h(P)=2|R|+2$ )

$$
\begin{aligned}
h\left(P \pm P_{0}\right) & \leq \max \left(\frac{4}{3} h(P)-\frac{8}{3}+2|t|, h(P)+2-2|t|\right) \\
& \leq \max \left(\frac{4}{3} h(P)-\frac{2}{3}, h(P)+2\right) .
\end{aligned}
$$

The result follows from (40), (41), (45).

Putting together Lemmas 3, 4, 5, 6 gives the following.

THEOREM 7. For a choice of sign,

$$
h\left(P \pm P_{0}\right) \leq \max \left(\frac{4}{3} h(P)+4, \min \left(\frac{4}{3} h(P)+6,2 h(P)\right)\right) .
$$

$\S 4$.

It is necessary now to obtain an estimate for $h(2 P)$.

THEOREM 8.

$$
h(2 P) \geq 4 h(P)-6
$$


Proof. As above, let $P=\left(\Delta R^{2} / S^{2}, \Delta R T / S^{3}\right)$, where

$$
\Delta R^{4}+m R^{2} S^{2}+\frac{n}{\Delta} S^{4}=T^{2} .
$$

Again, we assume that $\Delta$ is at most quadratic by adding to $P$, if necessary, the point $(0,0)$.

The duplication formula on $E$ gives $2 P=\left(x_{2 P}, y_{2 P}\right)$ where

$$
x_{2 P}=\frac{1}{4 R^{2} S^{2} T^{2}}\left(\Delta R^{4}-\frac{n}{\Delta} S^{4}\right)^{2} ;
$$

and in order to estimate $h(2 P)$ it is necessary to know what polynomial factors can be common to both numerator and denominator at (47). In fact, they are co-prime in $C[u]$, as may be seen as follows.

(i) Suppose there exists $\pi \in C[u], \pi \notin C$, such that

$$
\left(\Delta R^{4}-\frac{n}{\Delta} S^{4}, R\right) \equiv 0 \bmod \pi \text {. }
$$

Then $(n / \Delta, R) \equiv 0 \bmod \pi$, so that from $(46), T \equiv 0 \bmod \pi$, and $(n / \Delta) S^{4} \equiv$ $0 \bmod \pi^{2}$. Since $S \neq \equiv 0 \bmod \pi$, then $n \equiv 0 \bmod \pi^{2}$ which is impossible from (5).

(ii) Suppose there exists $\pi \in C[u], \pi \notin C$, such that

$$
\left(\Delta R^{4}-\frac{n}{\Delta} S^{4}, S\right) \equiv 0 \bmod \pi \text {. }
$$

Then $\Delta \equiv 0 \bmod \pi, T \equiv 0 \bmod \pi, \Delta \equiv 0 \bmod \pi^{2}$, impossible.

(iii) Suppose there exists $\pi \in C[u], \pi \notin C$, such that

$$
\left(\Delta R^{4}-\frac{n}{\Delta} S^{4}, T\right) \equiv 0 \bmod \pi
$$

Consider the following identity:

$$
\begin{gathered}
R^{2} T^{2}\left(3 \Delta^{3} R^{6}-m \Delta^{2} R^{4} S^{2}-5 n \Delta R^{2} S^{4}+2 m n S^{6}\right) \\
-\left(3 \Delta^{2} R^{4}+2 m \Delta R^{2} S^{2}-\left(m^{2}-4 n\right) S^{4}\right)\left(\Delta R^{4}-\frac{n}{\Delta} S^{4}\right)^{2} \\
=\left(m^{2}-4 n\right)\left(\frac{n}{\Delta}\right)^{2} S^{12} .
\end{gathered}
$$

It follows that $\left(m^{2}-4 n\right)(n / \Delta)^{2} S^{12} \equiv 0 \bmod \pi^{2}$; so in virtue of (5), and (ii) above, 


$$
\frac{n}{\Delta} \equiv 0 \bmod \pi
$$

But then from (48), $\Delta R^{4} \equiv 0 \bmod \pi$, so by (i) above,

$$
\Delta \equiv 0 \bmod \pi \text {. }
$$

And (50), (51) imply $n \equiv 0 \bmod \pi^{2}$, contradicting (5). Hence from (47),

$$
h(2 P)=\max \left\{\left|\left(\Delta R^{4}-\frac{n}{\Delta} S^{4}\right)^{2}\right|,\left|R^{2} S^{2} T^{2}\right|\right\} .
$$

Case I $|\Delta|+4|R|>4-|\Delta|+4|S|$ (i.e. $|\Delta|+2|R|>2|S|+2$ ).

Then $\left|\Delta R^{4}\right|>\left|\frac{n}{\Delta} S^{4}\right|,\left|\Delta R^{4}\right|>\left|m R^{2} S^{2}\right|$

so that $\left|\left(\Delta R^{4}-\frac{n}{\Delta} S^{4}\right)^{2}\right|=2|\Delta|+8|R|$,

and $2|T|=|\Delta|+4|R|$ whence $\left|R^{2} S^{2} T^{2}\right|=|\Delta|+6|R|+2|S|$.

Since $2|\Delta|+8|R|>|\Delta|+6|R|+2|S|$, then

$$
h(2 P)=2|\Delta|+8|R|=4 h(P)-2|\Delta| .
$$

Case II $|\Delta|+4|R|<4-|\Delta|+4|S|$.

As above, $\left|\left(\Delta R^{4}-\frac{n}{\Delta} S^{4}\right)^{2}\right|=8-2|\Delta|+8|S|$,

$$
\left|R^{2} S^{2} T^{2}\right|=4-|\Delta|+2|R|+6|S| \text {. }
$$

It follows that

$$
h(2 P)=8-2|\Delta|+8|S|=4 h(P)+8-2|\Delta| .
$$

Case III $|\Delta|+4|R|=4-|\Delta|+4|S|$.

Estimating the degrees of the polynomials occurring in the identity (49) gives

$$
\begin{aligned}
& \left|R^{2} T^{2}\left(3 \Delta^{3} R^{6}-m \Delta^{2} R^{4} S^{2}-5 n \Delta R^{2} S^{4}+2 m n S^{6}\right)\right| \leq\left|R^{2} T^{2}\right|+6|S|+6 \\
& \begin{aligned}
\left|\left(3 \Delta^{2} R^{4}+2 m \Delta R^{2} S^{2}-\left(m^{2}-4 n\right) S^{4}\right)\left(\Delta K^{4}-\frac{n}{\Delta} S^{4}\right)^{2}\right| \\
\quad \leq\left|\left(\Delta R^{4}-\frac{n}{\Delta} S^{4}\right)^{2}\right|+4|S|+4
\end{aligned} \\
& \text { and }\left|\left(m^{2}-4 n\right)\left(\frac{n}{\Delta}\right)^{2} S^{12}\right|=12-2|\Delta|+12|S|
\end{aligned}
$$

Consequently, either $\left|R^{2} S^{2} T^{2}\right|+6|S|+6 \geq 12-2|\Delta|+14|S|$ 
or $\left|\left(\Delta R^{4}-\frac{n}{\Delta} S^{4}\right)^{2}\right|+4|S|+4 \geq 12-2|\Delta|+12|S|$.

Thus either $\left|R^{2} S^{2} T^{2}\right| \geq 6-2|\Delta|+8|S|$

or $\left|\left(\Delta R^{4}-\frac{n}{\Delta} S^{4}\right)^{2}\right| \geq 8-2|\Delta|+8|S|$.

In any event, from (52),

$$
h(2 P) \geq 6-2|\Delta|+8|S|=4 h(P)-2|\Delta|-2 .
$$

Since $0 \leq|\Delta| \leq 2$, then (53), (54), (55) imply the result.

$\S 5$.

It is now possible to prove that (s) itself has a system of generators which are at most quadratic points of $E$.

Given $P \in \mathbb{S}$, assume as before that $P$ belongs to $\Delta$ where $\Delta$ is at most quadratic. Take $P_{0} \in \mathbb{S}, P_{0}$ at most quadratic, with $P \equiv P_{0} \bmod 2 S^{\prime}$. Let $P \pm P_{0}=2 Q$ where the sign is determined to satisfy the inequality of Theorem 7 .

Using Theorems 7, 8:

$$
4 h(Q)-6 \leq h(2 Q) \leq \max \left(\frac{4}{3} h(P)+4, \min \left(\frac{4}{3} h(P)+6,2 h(P)\right)\right)
$$

so that

$$
h(Q) \leq \max \left(\frac{1}{3} h(P)+\frac{5}{2}, \min \left(\frac{1}{3} h(P)+3, \frac{1}{2} h(P)+\frac{3}{2}\right)\right) .
$$

Suppose now $h(P) \geq 10$; then (56) gives

$$
h(Q) \leqq \frac{1}{3} h(P)+3<h(P) .
$$

Suppose

$$
h(P)=6,8 \text {; then }(56) \text { gives }
$$

$$
h(Q) \leq \frac{1}{2} h(P)+\frac{3}{2}<h(P) .
$$

Suppose

$$
h(P)=4 \text {; then }(56) \text { gives }
$$

$$
h(Q) \leq \frac{1}{3} h(P)+\frac{5}{2}<h(P) .
$$

Thus $h(Q)<h(P)$ for $h(P) \geq 4$. Inductively repeating the process, it is now evident that $B S$ is indeed generated by points which are at most quadratic. 
$\S 6$.

By noting the argument in Section 2 for deriving a system of generators for $\mathbb{S} / 2 \mathbb{S}$, it may be shown that Lemma 1 can be replaced by the stronger form:

Lemma 1'. The surface (2) has Mordell-Weil rank 4 over $C(u)$ if and only if the following hold:

(i) $n$ and $m^{2}-4 n$ are of degree 3 or 4

(ii) $n$ and $m^{2}-4 n$ are squarefree elements of $C[u]$.

Theorem 2, with a slightly modified proof, is still valid, as are Therems 7, 8 (N.B. Case III of Theorem 8, namely $\left|\Delta R^{4}\right|=\left|(n / \Delta) S^{4}\right|$ cannot occur when $|n|=3$ ). Accordingly, the group \&S is still generated by points at most quadratic.

TheOREM 9. The group of $C(u)$ rational sections of the elliptic surface $y^{2}=x\left(x^{2}+m x+n\right)$ where $m, n$ satisfy (3), (57), (58) has rank 4 and is generated by the $C(u)$ rational sections which are at most quadratic.

\section{$\S 7$.}

Since $(5)$ is finitely generated over $C(u)$, there exists an algebraic number field $K_{0}$ such that $K_{0}(u)$ is the least field over which \&s is defined. $K_{0}$ is called the field of definition of \&s, and is interesting in its own right because of applications to the Néron-Severi group of $E$. See Swinnerton-Dyer [8] who considers this problem more generally.

$K_{0}$ for the examples of this paper may have large degree over $\boldsymbol{Q}$, although crude estimates from the given construction will show that $\left[K_{0}: Q\right]$ is bounded above by $24^{8}$.

In any given instance, it is straightforward to determine $K_{0}$ since it suffices to find the field of definition of the points of the curve which are at most quadratic.

As an example, consider the curve

$$
y^{2}=x\left(x^{2}-8 x-12\left(u^{4}-1\right)\right) .
$$

Following arguments of Section 2, it is readily found that $K_{0}$ for (59) is simply the splitting field of $n$ and $m^{2}-4 n$ at (2), namely

$$
K_{0}=Q(\sqrt{-1}, \sqrt{2}, \sqrt[4]{3})
$$


Schwartz [6] finds a family of elliptic surfaces of rank 4, with corresponding $K_{0}$ equal to $\boldsymbol{Q}$.

\section{REFERENCES}

[1 ] B. J. Birch, H. P. F. Swinnerton-Dyer, Notes on elliptic curves II, J. reine angew. Math., 218 (1965), 79-108.

[2] J. W. S. Cassels, W. J. Ellison, A. Pfister, On sums of squares and on elliptic curves over function fields, J. Number Theory, 3 (1971), 125-149.

[ 3 ] D. A. Cox, S. Zucker, Intersection numbers of sections of elliptic surfaces, Invent. Math., 53 (1979), 1-44.

[ 4 ] K. Kodaira, On compact analytic surfaces III, Ann. of Math., 78 (1963), 1-40.

[ 5 ] C. F. Schwartz, A Mordell-Weil group of rank 8 and a subgroup of finite index, Nagoya Math. J., 93 (1984), 19-26.

[6] _ - On a family of elliptic surfaces with mordell-Weil rank 4, to appear.

[ 7 ] T. Shioda, On elliptic modular surfaces, J. Math. Soc. Japan, 24 (1972), 20-57.

[8] H. P. F. Swinnerton-Dyer, The field of definition of the Néron-Severi group, Studies in Pure Mathematics, Hung. Acad. of Sci., Turan Memorial Volume, 1983.

Arizona State University

Department of Mathematics

Tempe, Arizona 85287 